\section{High performance surgical handpieces}

Optimal cooling and illumination of the oral cavity is essential to delivering effective treatment, which is why $\mathrm{W} \& \mathrm{H}$ offers innovative straight and contra-angle surgical handpieces, some of which feature their own independent power supply and integrated LED+ illumination.

These advanced solutions are ideal for use in oral and maxillofacial surgery, and implantology.

Each handpiece facilitates fatigue-free operations due to their highly ergonomic design, which is manufactured from high quality stainless steel with a scratch-resistant coating to ensure durability.

Designed to complement modern decontamination protocols, practitioners can rest assured they are able to maintain the high performance of W\&H handpieces for a particularly long service life.

More information is available by visiting www.wh.com/en_uk, calling 01727874990 or emailing office.uk@wh.com.

\section{Masterclass on treatment of edentulous patients}

Dental professionals are being invited to attend what is described as a comprehensive masterclass on the treatment of endentulous patients with dental implants.

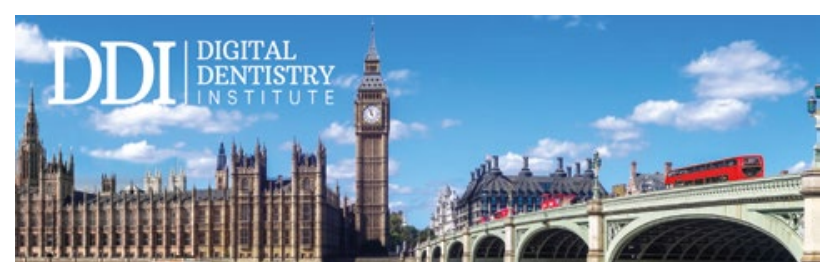

The Digital Dentistry Institute is holding a conference on 4 and 5 July 2019 in London, entitled Full Arch Rehabilitation With Dental Implants - A Digital and Analogue Approach.

The event is being presented by the editor of the $B D J$ Clinician's Guide book 'Graftless solutions for the edentulous patient' (https://shop.bda.org/graftless-solutions) - Dr Saj Jivraj, Dr Bobby Birdi, and Dr Sundeep Rawal.

The event's topics will include:

1. Diagnosis and treatment planning of the edentulous patient

2. The use of digital technologies to simplify workflow

3. Surgical protocols - guided vs non-guided

4. Restorative protocols for predictability - space, occlusion, material

5. Clinical checklists

6. Immediate loading direct and indirect approaches

7. Materials for definitive restorations

8. CADCAM technology

9. Laboratory procedures

10. Maintenance protocols

11. Managing complications

12.Implementing systems in your practice.

To register for the event, call DDI UK on 01344291744 or visit https://ddidental-programs.azurewebsites.net/london.html.

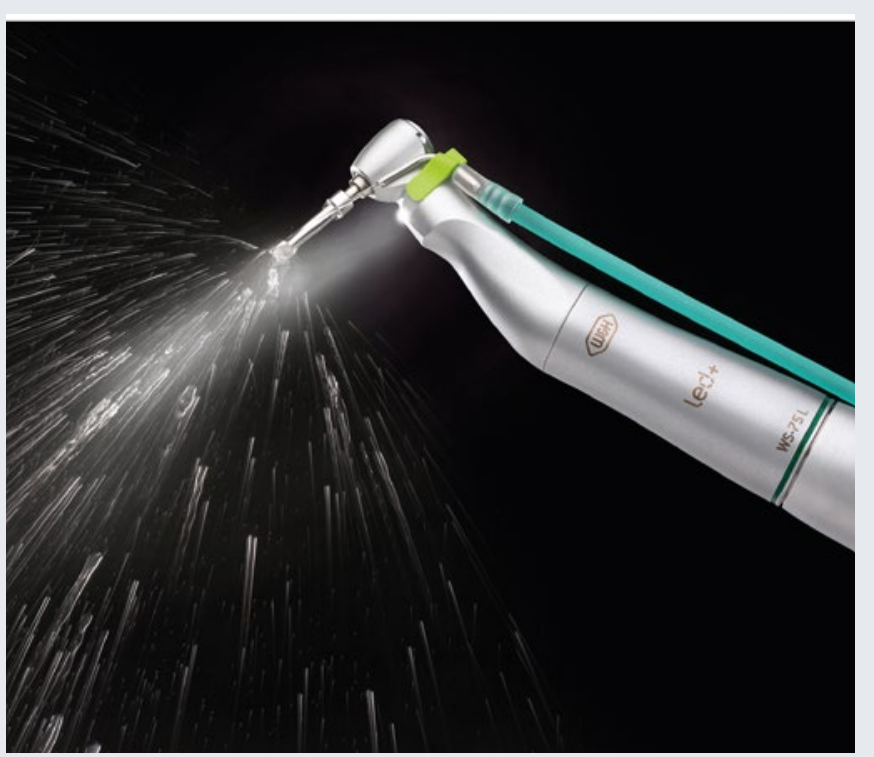

\section{Clean water without chemicals}

Dental treatment systems offer ideal conditions under which biofilm can form and micro-organisms such as pseudomonas, legionella and cryptosporidium can flourish.

These micro-organisms can be exposed to the patient via the cooling water, mouth rinsing water and aerosol exposure.

Therefore, Hygowater from Dürr Dental - a system that promises safe and reliable service water processing - is the answer.

Hygowater ensures the service water in your practice always meets the same stringent requirements as drinking water. This standard is consistent with the advice given by the Robert Koch Institute.

Water-carrying systems in treatment units can still, however, harbour various micro-organisms, which can colonise and form a biofilm which adheres to the inner walls of the unit. To ensure optimum safety, micro-organisms must be reduced to a minimum and biofilm permanently removed from hoses and pipes.

The Hygowater system fulfils all the legal requirements for water hygiene as well as satisfying the meticulous standards demanded by the German Drinking Water Ordinance as well as meeting the requirements for a Class I medical device.

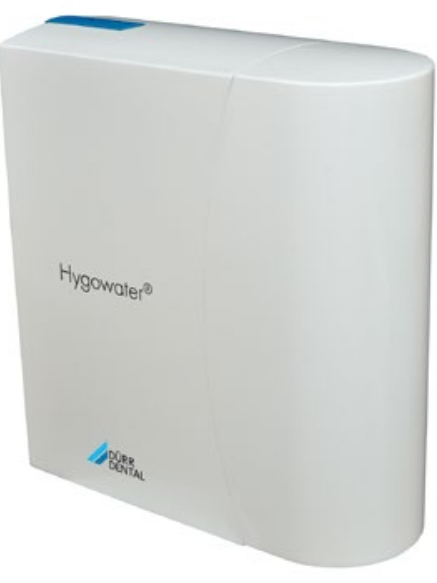

The compact unit is extremely easy to operate. The unique combination of filtration and electrolysis prevent biofilm formation and thus minimises infection risks to both patients and practitioners. As well as being good for the safety of the practice, it is also good for the environment, as long-term drinking water quality is ensured without the use of any chemical additives.

More information is available at https://www.duerrdental.com/ en/products/hygiene/hygiene-appliances/water-treatment-systems/ water-treatment-systems-for-treatment-units/hygowater/. 